\title{
Degludec is superior to glargine in terms of daily glycemic variability in people with type 1 diabetes mellitus
}

\author{
Chiho Yamamoto $^{1)}$, Hideaki Miyoshi ${ }^{1)}$, Yutaka Fujiwara ${ }^{2)}$, Reina Kameda ${ }^{1)}$, Mei Ichiyama ${ }^{1)}$, \\ Hiroshi Nomoto $^{1)}$, Hiraku Kameda ${ }^{1)}$, Akinobu Nakamura ${ }^{1)}$ and Tatsuya Atsumi ${ }^{1)}$ \\ 1) Division of Rheumatology, Endocrinology and Nephrology, Hokkaido University Graduate School of Medicine, Sapporo 060-8638, \\ Japan \\ 2) KKR Sapporo Medical Center, Sapporo 062-0931, Japan
}

\begin{abstract}
To investigate the differences in glycemic variability between the long-acting insulins glargine and degludec using continuous glucose monitoring, we conducted an open-label, multicenter, prospective, observational study that enrolled 21 participants with type 1 diabetes mellitus currently receiving basal-bolus insulin therapy with glargine. To avoid the potential influence of diet and exercise on glycemic control, all participants were housed and monitored within the hospital for the duration of the study. Once glycemic control was achieved with glargine, glycemic variability was evaluated using continuous glucose monitoring for 3 days. Glargine was then replaced by degludec and glycemic variability again assessed via continuous glucose monitoring. The primary outcome measure of mean amplitude of glycemic excursions was significantly reduced with degludec $(p=0.028)$, as was area under the curve for daily blood glucose level $<70 \mathrm{mg} / \mathrm{dL}(p=0.046)$. The required insulin dose was reduced up to $25 \%$ in the degludec group, although $24-\mathrm{h}$ mean glucose concentrations were not different between groups. In conclusion, once or twice daily glargine was successfully replaced by a daily injection of degludec. When replacing glargine with degludec, a lower dose should be utilized to avoid hypoglycemia. Degludec is an effective and promising long-acting insulin that reduced hypoglycemia and daily blood glucose variability in participants with type 1 diabetes.
\end{abstract}

Key words: Continuous glucose monitoring, Insulin degludec, Type1 diabetes mellitus

INSULIN GLARGINE (Gla) is currently the most commonly used long-acting (basal) insulin analog. Recently, insulin degludec (Deg) has been approved as a new long-acting insulin and is now available for daily clinical practice. Deg has longer and flatter effects compared to other conventional long-acting insulins [1]. The durability of circulating Gla is less than $24 \mathrm{~h}$ $[2,3]$, thus a subset of people with insulin-deficient type 1 diabetes mellitus must receive twice-daily injections of Gla. Additionally, it was reported that glycemic control can be improved in people with type 1 diabetes mellitus by administering Gla twice daily as compared to a single daily dose [3]. In contrast, a single injection of Deg had an evenly distributed glucose lowering effect

Submitted Jul. 25, 2015; Accepted Oct. 1, 2015 as EJ15-0438 Released online in J-STAGE as advance publication Oct. 31, 2015 Correspondence to: Hideaki Miyoshi, M.D., Ph.D., Division of Rheumatology, Endocrinology and Nephrology, Hokkaido University Graduate School of Medicine, North 15, West 7, Kitaku, Sapporo 060-8638, Japan. E-mail: hmiyoshi@med.hokudai.ac.jp

(C) The Japan Endocrine Society over a $24 \mathrm{~h}$ period with a duration of action greater than $26 \mathrm{~h} \mathrm{[4].} \mathrm{The} \mathrm{reduced} \mathrm{number} \mathrm{of} \mathrm{insulin} \mathrm{injections} \mathrm{is}$ thought to be preferred by people requiring insulin and is considered an improvement in quality of life [5].

Although the phase 3 trials of Deg showed good performance as a new basal insulin in people with type 1 diabetes [6-8], few studies have shown the effect of Deg on the stability of glucose levels using continuous glucose monitoring (CGM) $[9,10]$. CGM systems allow visualization of the daily variations in blood glucose levels, facilitating the detection of postprandial hyperglycemia and asymptomatic hypoglycemia. Therefore, these systems can provide a more accurate means to evaluate the efficacy of glucose lowering therapies within individual participants [11]. Blood glucose variability is an important contributing factor to the severity of coronary artery disease independent of $\mathrm{HbA} 1 \mathrm{c}[12$, 13], as well as an independent predictor of mortality [14]. Mean amplitude of glycemic excursions (MAGE) is a marker of daily blood glucose variability relating to higher postprandial blood glucose or hypoglycemia, 
and was recently reported to correlate closely with cognition level and oxidative stress in vivo $[15,16]$. It is worth evaluating the difference in daily blood glucose variability between Deg and Gla using CGM systems.

In a phase 3 clinical trial, Deg decreased the frequency of nocturnal hypoglycemia compared to Gla [6-8]. However, hypoglycemia was defined as when the participants experienced hypoglycemic symptoms and blood glucose levels were less than $56 \mathrm{mg} / \mathrm{dL}$ or when severe hypoglycemia was present that required assistance and therefore, asymptomatic hypoglycemia was not included. Previous CGM data indicate that asymptomatic hypoglycemia is unexpectedly common among people with type 1 diabetes mellitus [17]. Furthermore, in the phase 3 trials of Deg, basal insulin dose was titrated to target a fasting blood glucose of 70-89 mg/dL. People with type 1 diabetes mellitus are more susceptible to hypoglycemia because of the severe fluctuation in their blood glucose. Therefore the lower blood glucose target in these trials could increase the frequency of hypoglycemia as compared to that observed in standard practice. The goal of the present study was thus to investigate the superiority of Deg in terms of glycemic variability compared with Gla under conditions more representative of clinical practice (less than $130 \mathrm{mg} / \mathrm{dL}$ at premeal and less than $180 \mathrm{mg} / \mathrm{dL}$ at 2-hours (h) postprandial) and to assess incidence of asymptomatic hypoglycemia via CGM. In order to minimize the potential influence of factors such as diet and exercise on glycemic control, and compare the intrinsic efficacy of the two basal insulins, all CGM data were collected from people with type 1 diabetes mellitus that were hospitalized and following optimization of glycemic control.

\section{Materials and Methods}

\section{Participants}

This open-label, multicenter, comparative, observational, prospective study enrolled people with type 1 diabetes who had been admitted to Hokkaido University Hospital or KKR Sapporo Hospital. All participants received an explanation of the study procedures and potential risks associated with participation and gave their written informed consent prior to entry. The inclusion criteria were hospitalized people with type 1 diabetes over the age of 20 receiving basal-bolus insulin therapy including Gla as basal insulin. People who had unstable retinopathy of diabetes, a history of anaphylaxis to Deg, were pregnant women, had a persistent elevation of their serum transaminase, or had renal dysfunction were excluded. This study was approved by the Institutional Review Board of Hokkaido University Hospital and was performed in accordance with the Declaration of Helsinki.

\section{Design of the study}

Enrolled participants were provided a fixed caloric diet that was calculated to adequately meet the nutritional requirements of each participant. The participants were asked to avoid excessive exercise and snacking. Minimal glucose consumption was allowed only when symptomatic hypoglycemia occurred. This study was performed using the following protocol (Fig. 1).

Glycemic control was targeted at less than $130 \mathrm{mg} /$ $\mathrm{dL}$ for average premeal blood glucose levels and less than $180 \mathrm{mg} / \mathrm{dL}$ for average 2-h postprandial glucose levels, according to the Japan Diabetes Society guidelines. The insulin doses administered were adjusted by expert endocrinologists in order to ensure optimum glycemic control. Administration of other antidiabetic medications during the study period was not allowed. Once glycemic control met the target criteria described above, glycemic variability was evaluated by CGM for 3 days (Day 1-3). Following this 3 day monitoring period, the basal insulin was switched from Gla to Deg. The initial dose of Deg that was administered was $20 \%$ less than the dose of Gla the participants had been receiving. The next 3 days (Day 4-7) were used to optimize the dose of bolus insulin and Deg to achieve the same target of glycemic control, and CGM data were then collected for 3 days (Day 7-9). Fasting blood samples were taken before and after the study.

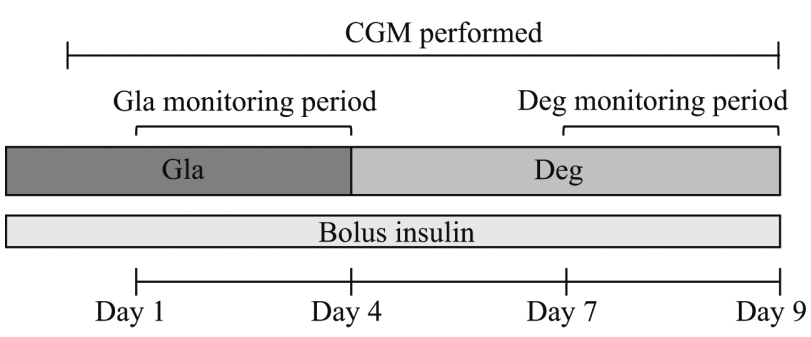

Fig. 1 Study design. The enrolled hospitalized participants with type 1 diabetes were treated and got the target range of glucose using CGM as described in the text. After 3 days monitoring (day 1-3) using Gla, Gla was replaced to Deg at $20 \%$ lower dose than Gla. Insulin dose was adjusted during day 4-6, then the last 3 days monitoring (day 7-9) was done using Deg. CGM, continuous glucose monitoring; Gla, glargine; Deg, degludec. 
After the study, the participants were asked to select which basal insulin they preferred and allowed to continue use of the insulin they had selected.

\section{CGM}

CGM was performed using the CGMS-Gold $₫$ device manufactured by Medtronic Inc. A 5-10-min delay occurs with CGMS Gold in the glucose measurement as it involves the use of interstitial fluid, compared with methods involving the use of venous whole blood. It is also reported to be less accurate in glucose measurement during hypoglycemia [18]. The interstitial glucose values obtained with CGMS Gold were adjusted using self-monitored blood glucose values collected four times a day via finger prick and using a handheld glucometer. Blood glucose values indicated by CGMS Gold were virtually identical to the venous values [19].

\section{Glycemic variability}

"Nighttime" was defined as $0000 \mathrm{~h}$ to $0600 \mathrm{~h}$ and "early morning," as $0300 \mathrm{~h}$ to $0600 \mathrm{~h}$. In this study, "hypoglycemia" was defined as the area under the curve (AUC) <70 mg/dL; and "hyperglycemia," as the area under the curve (AUC) $>180 \mathrm{mg} / \mathrm{dL}$.

The primary outcome was glycemic variability (MAGE) measured by CGM over a 24-h period for 3 consecutive days (study days 1-3 for Gla and 7-9 for Deg). Secondary endpoints included the SD values during 24-h glucose levels, hypoglycemia over a 24-h period, hypoglycemia during nighttime, hypoglycemia during daytime, hyperglycemia during a 24-h period, mean of daily difference (MODD) for a 24-h period, MODD during early morning, as well as changes in the dose of insulin administered. MODD has been used as an index of day-to-day glucose variability [20].

\section{Statistical analysis}

Results are expressed as mean $\pm \mathrm{SD}$. A paired $t$-test was employed to compare treatment differences between Gla and Deg. A $p$-value $<0.05$ was considered statistically significant. Data were analyzed using Ekuseru-Toukei 2012 (Social Survey Research Information, Tokyo, Japan).

\section{Results}

\section{Participant characteristics}

A total of 21 hospitalized people with type 1 diabetes were enrolled in the study ( 5 men, 16 women).
Participant baseline data were as follows: age, $55 \pm 11$ years; duration of disease, $13.9 \pm 8.4$ years; glycosylated hemoglobin $(\mathrm{HbAlc}), 67 \pm 4 \mathrm{mmol} / \mathrm{mol}(8.3 \pm$ $2.5 \%)$; body mass index, $21.3 \pm 3.4 \mathrm{~kg} / \mathrm{m}^{2}$; total daily insulin dose, $0.67 \pm 0.24 \mathrm{U} / \mathrm{kg}(36.2 \pm 12.8 \mathrm{U})$; total daily basal insulin dose, $0.24 \pm 0.08 \mathrm{U} / \mathrm{kg}(13.2 \pm 5.6$ $\mathrm{U})$; the ratio of basal insulin to total daily insulin, 37.6 $\pm 10.7 \%$. Thirteen participants were receiving Gla twice daily and the others were receiving a single daily injection. The ratio of morning to evening basal insulin dose was approximately 10:9 in participants receiving Gla twice daily (Table 1).

The insulin preparation used for preprandial bolus supplementation was either insulin aspart, insulin lispro, or insulin glulisine. Insulin secretion in 17 participants was completely defective and serum CPR was undetectable, while the other 4 participants had CPR concentrations that averaged $0.48 \pm 0.17 \mathrm{ng} / \mathrm{mL}$. Six of 16 women were pre-menopausal, but none of them were menstruating during the study. When glycemic control met the predefined criteria prior to initiating the study, the average premeal blood glucose levels were $129.4 \pm 35.2 \mathrm{mg} / \mathrm{dL}$ and the average $2 \mathrm{~h}$ postprandial glucose levels were $163.1 \pm 47.1 \mathrm{mg} / \mathrm{dL}$.

\section{Comparison of CGM findings (Table 2)}

Although thirteen participants were receiving Gla twice a day, a single daily injection of Deg was sufficient to control glucose levels in these participants. The primary endpoint of 24-h MAGE was significantly decreased from $144.4 \pm 56.6 \mathrm{mg} / \mathrm{dL}$ to $121.7 \pm 42.2$ $\mathrm{mg} / \mathrm{dL}(p=0.028)$ when Gla was replaced by Deg (Fig. 2a), while no significant change was observed in 24-h mean glucose levels $(153.9 \pm 31.8 \mathrm{mg} / \mathrm{dL}$ and $153.6 \pm$ $26.2 \mathrm{mg} / \mathrm{dL}$ respectively, $p=0.959)$. Early morning

Table 1 Participants demographics and baseline characteristics

\begin{tabular}{|c|c|}
\hline & Type1 DM \\
\hline $\mathrm{N}$ & 21 \\
\hline Sex (men/women) & $5 / 16$ \\
\hline Age (years) & $55 \pm 11+$ \\
\hline Duration of disease (years) & $13.9 \pm 8.4 *$ \\
\hline $\begin{array}{l}\mathrm{HbA} A \mathrm{c}(\mathrm{mmol} / \mathrm{mol}) \\
(\%)\end{array}$ & $\begin{array}{c}67 \pm 4 \stackrel{\ddagger}{\ddagger} \\
8.3 \pm 2.5 \ddagger\end{array}$ \\
\hline $\operatorname{BMI}\left(\mathrm{kg} / \mathrm{m}^{2}\right)$ & $21.3 \pm 3.4+$ \\
\hline Total daily insulin dose (U/kg) & $0.67 \pm 0.24 *$ \\
\hline Total daily Gla dose (U/kg) & $0.24 \pm 0.08 *$ \\
\hline $\begin{array}{l}\text { The number of basal insulin injection } \\
\text { (twice/once a day) }\end{array}$ & $13 / 8$ \\
\hline
\end{tabular}

$\$$ Values are expressed as means \pm SD. 
Table 2 Comparison of 3 days' CGM data of 21 participants between Gla and Deg

\begin{tabular}{lccc}
\hline & Gla & Deg & $p$ value \\
\hline 24-h mean glucose levels (mg/dL) & $153.9 \pm 31.8$ & $153.6 \pm 26.2$ & 0.959 \\
\hline MAGE (mg/dL) & & & \\
$\quad$ 24-h & $144.4 \pm 56.6$ & $121.7 \pm 42.2$ & 0.028 \\
$\quad$ early morning & $41.8 \pm 32.6$ & $28.7 \pm 11.4$ & 0.048 \\
\hline 24-h SD values glucose levels (mg/dL) & $51.6 \pm 18.1$ & $43.7 \pm 13.7$ & 0.031 \\
\hline AUC $<70$ (mg/dL·h) & & & \\
$\quad$ 24-h & $0.8 \pm 1.1$ & $0.2 \pm 0.5$ & 0.046 \\
$\quad$ Nighttime & $0.5 \pm 0.9$ & $0.2 \pm 0.3$ & 0.090 \\
Daytime & $0.6 \pm 1.0$ & $0.2 \pm 0.3$ & 0.040 \\
\hline 24-h AUC $>180$ (mg/dL·h) & $17.0 \pm 16.7$ & $14.0 \pm 13.6$ & 0.321 \\
\hline MODD (mg/dL) & & & \\
24-h & $50.2 \pm 16.2$ & $44.1 \pm 10.2$ & 0.084 \\
$\quad$ Early morning & $51.2 \pm 28.2$ & $38.7 \pm 18.0$ & 0.089 \\
\hline Insulin dose (U/kg) & & & \\
$\quad$ Twice a day & & & \\
$\quad$ Basal insulin & $0.27 \pm 0.10$ & $0.20 \pm 0.07$ & $<0.001$ \\
$\quad$ Bolus insulin & $0.48 \pm 0.28$ & $0.49 \pm 0.28$ & 0.785 \\
$\quad$ Once a day & $0.21 \pm 0.04$ & $0.15 \pm 0.05$ & 0.007 \\
$\quad$ Basal insulin & $0.38 \pm 0.11$ & $0.36 \pm 0.10$ & 0.609 \\
\hline Bolus insulin & & & \\
\hline
\end{tabular}

MAGE, mean amplitude of glycemic excursions; MODD, mean of daily difference; 24-h, all day; early morning, 3 to $6 \mathrm{am}$; nighttime, 0 to $6 \mathrm{am}$. Values are expressed as means $\pm \mathrm{SD}$.

(a)

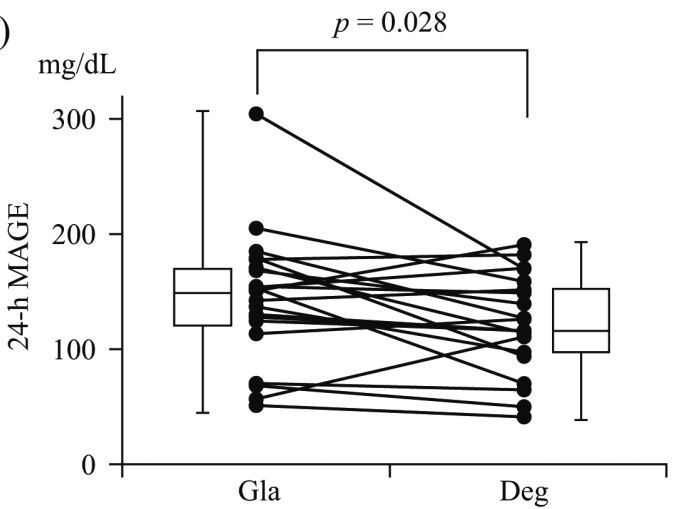

(c) $\mathrm{mg} / \mathrm{dL} \cdot \mathrm{h}$

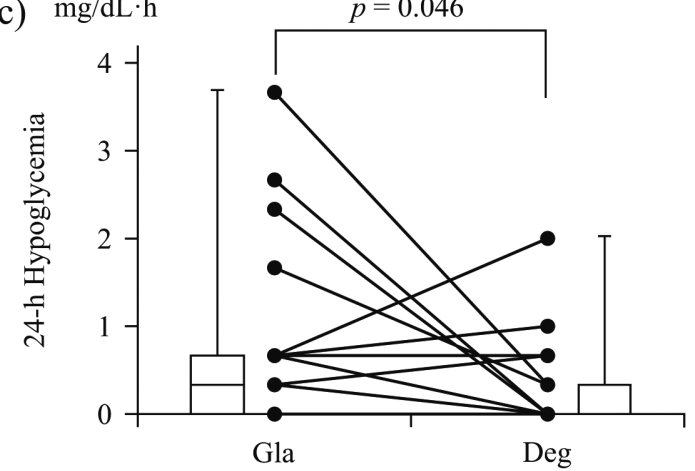

(b)

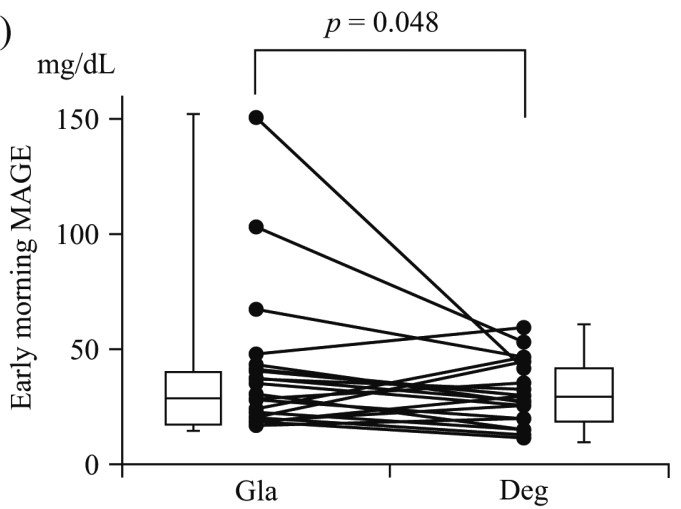

(d) $\mathrm{mg} / \mathrm{dL} \cdot \mathrm{h}$
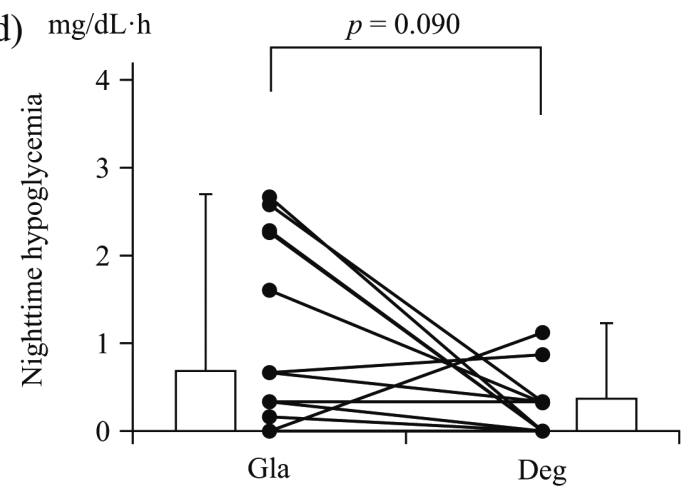

Fig. 2 Comparison of MAGE $(a, b)$ and hypoglycemia (c, d) between Gla and Deg. MAGE (a and b) and hypoglycemia, defined as an area under curve $<70 \mathrm{mg} / \mathrm{dL}$ (c and d), were calculated from 3 days' CGM data by each long-acting insulin. Early morning was defined during 3-6 a.m. Nighttime was defined during 0-6 a.m. Gla, glargine; Deg, degludec; MAGE, mean amplitude of glycemic excursions; CGM, continuous glucose monitoring 
MAGE was also significantly decreased from $41.8 \pm$ $32.6 \mathrm{mg} / \mathrm{dL}$ to $28.7 \pm 11.4 \mathrm{mg} / \mathrm{dL}$ ( $p=0.048$ ) (Fig. 2b). Similarly, the standard deviation of daily blood glucose concentrations was significantly decreased from $51.6 \pm 18.1 \mathrm{mg} / \mathrm{dL}$ to $43.7 \pm 13.7 \mathrm{mg} / \mathrm{dL}(p=0.031)$ by switching from Gla to Deg. Significant reductions in 24-h and daytime hypoglycemia were observed (Fig. $2 c$ ), while nighttime hypoglycemia only tended to decrease $(p=0.090)$ (Fig. 2d). The daytime hypoglycemia values were $0.6 \pm 1.0 \mathrm{mg} / \mathrm{dL} \cdot \mathrm{h}$ and $0.2 \pm 0.3 \mathrm{mg} /$ $\mathrm{dL} \cdot \mathrm{h}(p=0.040)$ for Gla and Deg, respectively.

A small, nonsignificant $(p=0.321)$ decrease in hyperglycemia (AUC $>180 \mathrm{mg} / \mathrm{dL}$ ) was observed between Gla $(17.0 \pm 16.7 \mathrm{mg} / \mathrm{dL} \cdot \mathrm{h})$ and to Deg $(14.0 \pm$ $13.6 \mathrm{mg} / \mathrm{dL} \cdot \mathrm{h})$ groups. MODD during early morning also had a trend to decrease with Deg from $51.2 \pm 28.2$ $\mathrm{mg} / \mathrm{dL}$ to $38.7 \pm 18.0 \mathrm{mg} / \mathrm{dL}(p=0.089)$.

\section{Insulin dose (Table 2)}

Previous phase 3 clinical trials using Deg reported a lower dose of Deg was required to achieve similar glucose lowering as Gla [6-8]. Therefore, in this trial, the initial dose of Deg provided when switching from Gla to Deg was $20 \%$ less than the Gla dose the participant had been receiving. Hypoglycemia was minimized using this dosing strategy and subsequent doses of Deg were adjusted accordingly. For participants who received Gla twice a day, their basal insulin dose was significantly reduced $(25.5 \%)$ with Deg from $0.27 \pm$ $0.10 \mathrm{U} / \mathrm{kg}$ to $0.20 \pm 0.07 \mathrm{U} / \mathrm{kg}(14.0 \pm 6.1 \mathrm{U}$ to $10.4 \pm$ $4.6 \mathrm{U})(p<0.001)$ (Fig. 3a), and bolus insulin dose was not changed $(0.48 \pm 0.28 \mathrm{U} / \mathrm{kg}$ to $0.49 \pm 0.28 \mathrm{U} / \mathrm{kg}(23.9$ $\pm 11.1 \mathrm{U}$ to $24.3 \pm 12.4 \mathrm{U})(p=0.597))$. Similarly, in the participants who received Gla once daily, their basal insulin dose was significantly reduced $(24.7 \%)$ with Deg from $0.21 \pm 0.04 \mathrm{U} / \mathrm{kg}$ to $0.15 \pm 0.05 \mathrm{U} / \mathrm{kg}(12.4$ $\pm 5.1 \mathrm{U}$ to $9.4 \pm 5.5 \mathrm{U})(p=0.007)$ (Fig. $3 \mathrm{~b})$, and bolus insulin dose was not changed $(0.38 \pm 0.11 \mathrm{U} / \mathrm{kg}$ to 0.36 $\pm 0.10 \mathrm{U} / \mathrm{kg}(21.2 \pm 8.7 \mathrm{U}$ to $20.0 \pm 8.9 \mathrm{U})(p=0.609))$.

\section{Discussion}

Deg is the third long-acting insulin available for daily clinical practice since March 2013 in Japan. Although this new insulin is expected to perform well in phase 3 studies [6-8], there is limited data when it comes to the careful evaluation of its efficacy using CGM. In this study, we used CGM to compare the blood glucose stabilizing effects between long-acting insulin Gla and Deg in people with type 1 diabetes mellitus.

Deg significantly improved MAGE as the primary outcome, and standard deviation of daily blood glucose compared with Gla in people with type 1 diabetes mellitus. Moreover, significant lowering of early morning
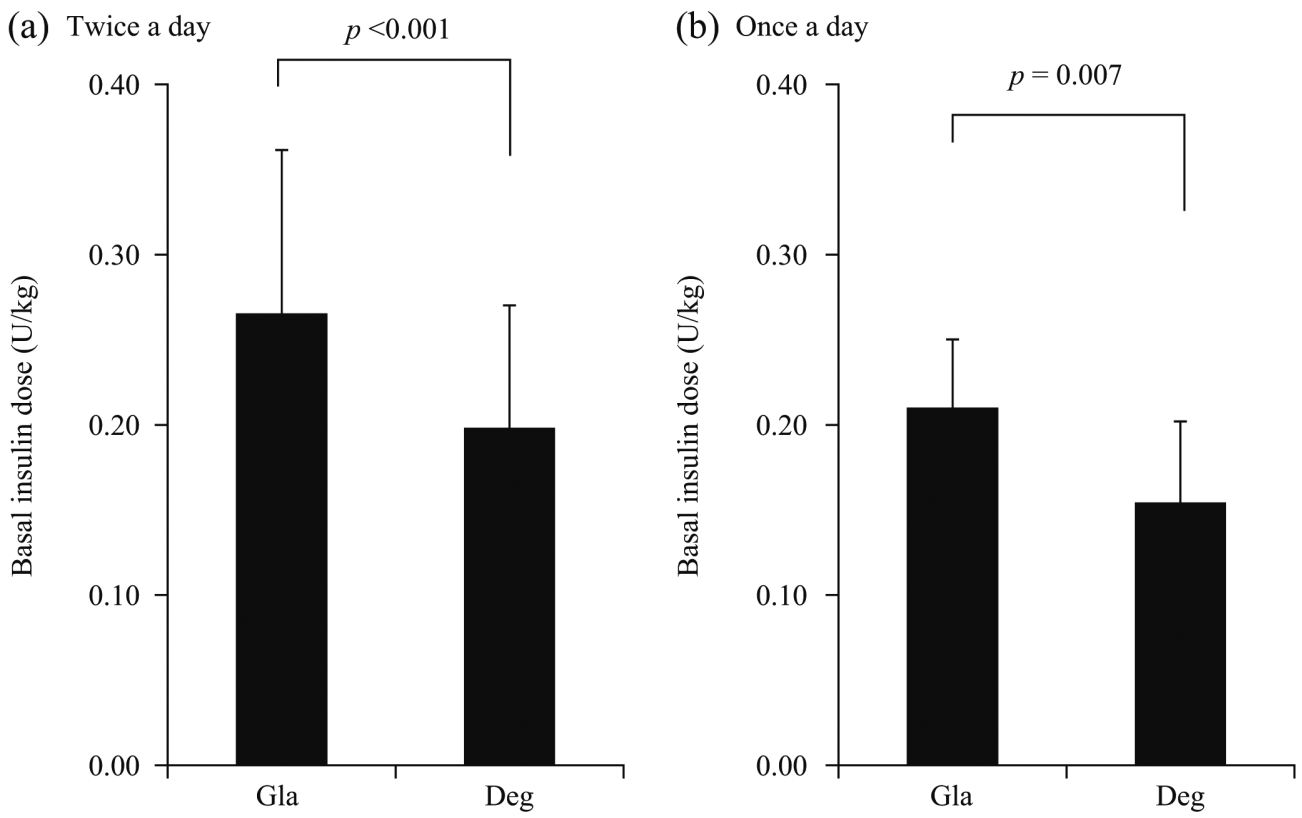

Fig. 3 Comparison of basal insulin dose between Gla and Deg in the patients who used Gla twice a day (a) or once a day (b) before switching insulin. Gla, glargine; Deg, degludec. 
MAGE during sleep is important for people with diabetes. Lower MAGE during the early morning leads to decreased risk of the somogyi effect or dawn phenomenon resulting in lower risk of hypoglycemia as well as hyperglycemia [21]. The longer duration of action, reduced peak, and lower variability in pharmacodynamic action of Deg may explain these observed results $[1,22,23]$. Moreover, lower risk of hypoglycemia during sleep would be beneficial for people with diabetes. Deg has a relatively low intra-subject pharmacodynamic variability in the steady state compared to Gla [1], thus the day-to-day variation of glucose (MODD) is expected to improve. However, there was no significant difference in early morning MODD $(p=0.089)$ in the present study. A sub-analysis was performed that included only the 17 participants whose insulin secretion was completely defective. In this population, a significant improvement in MODD both 24-h and early morning was seen with Deg $(p=0.022$ and 0.001, respectively). These data suggest that type 1 diabetic patients with complete insulin deficiency could receive more benefits in regulation of day-to-day variation in glucose levels by using Deg as their basal insulin.

Average daily blood glucose concentrations were similar between Gla and Deg. Given this lack of difference, replacement of Gla to Deg would not affect $\mathrm{HbA1c}$ values. This study was designed to assess glycemic variability after achieving targeted premeal and $2 \mathrm{~h}$ postprandial blood glucose levels with Gla or Deg. For this reason it is reasonable to assume that average blood glucose concentrations were similar despite differences in glucose variability. Consistent with the reports from Phase 3 trials, the dose of insulin Deg utilized in this trial was approximately $25 \%$ lower than that of Gla.

Recently, the same group has published two reports assessing the effects of Deg and Gla using CGM with a test diet $[9,10]$. Patients with type 1 diabetes were switched from Gla or detemir to Deg and followed for 12 or 24 weeks. Consistent with our findings, patients required a lower daily dose of Deg compared to their prior insulin dose and the required dose of basal insulin decreased significantly 24 weeks after switching. However, the improvement in MAGE that we report here was not observed in this previous study [10]. The reason for the discrepancy in CGM data is unclear, but may be due to the differences in the number of participants or the inclusion of hospitalized patients in the current study whose diet and activity were closely monitored. The pharmacokinetic profile of Deg is lon- ger and flatter than that of Gla [1]. Following a single injection, Deg remains detectable in circulation for more than $26 \mathrm{~h}$ [4], while Gla is no longer detectable by $24 \mathrm{~h}[2,3]$. As the duration of action of Deg is greater this results in an accumulation of Deg over time and decreases the required daily dose to maintain glucose levels. Our results confirmed the necessity to modify insulin dose when making Deg dosing calculations based on insulin Gla action.

Interestingly, phase 3 trials have reported that Deg lowers the frequency of nocturnal hypoglycemia [6-8] while our data showed a lower risk of daytime but not nocturnal hypoglycemia. There are several plausible explanations for this discrepancy. First, the targeted fasting blood glucose levels were more strictly defined (79-90 mg/dL) in the phase 3 trials [6-8] which would increase the likelihood of nocturnal hypoglycemic events. Second, only symptomatic and self-monitored blood glucose levels under $56 \mathrm{mg} / \mathrm{dL}$ were reported as hypoglycemia in the phase 3 trials, while the definition in the current study was any blood glucose value under $70 \mathrm{mg} / \mathrm{dL}$ using CGM. Using CGM Chico et al. reported that $62.5 \%$ of people experienced hypoglycemia unawareness suggesting that many asymptomatic hypoglycemia events highly likely went undetected during the phase 3 studies [17]. In addition, phase 3 trials are conducted as outpatient studies, while this inpatient study was conducted under the constant supervision of hospital staff and tightly controlled for diet and exercise. The intensive nature of this study design provided a means to accurately evaluate the intrinsic efficacy of each basal insulin while minimizing potential confounding variables.

The potential limitations of this study include the small sample size and short study duration. Additionally, this study was not conducted as a crossover study, as this would increase the required hospitalization of participants due to the additional washout period requirements thereby increasing the difficulty of enrollment. Since Gla was used ahead of Deg in the evaluation period, it is possible that this was advantageous to Deg. Subsequent studies using a more robust study design, with a larger population and conducted over a longer period of time would likely verify and extend the findings of the current study.

In conclusion, people with type 1 diabetes receiving once or twice daily injections of Gla were transitioned to a once daily injection of Deg which was associated with a dose reduction of approximately $25 \%$. 
Once an optimum dose of Deg was determined Deg reduced hypoglycemia and daily blood glucose variability (MAGE and SD) which could provide an additional benefit of decreasing cardiovascular events.

\section{Author Contribution}

C.Y. contributed to the data analysis and wrote the manuscript. H.M. and A.N. contributed to discussion, reviewed and edited the manuscript. H.M. designed and performed the research, and wrote the manuscript.

\section{Acknowledgements}

H.M. thanks Dr. James W. Perfield II (University of Missouri, MO, USA) for his continued support and mentorship.

\section{Disclosure Statement}

No potential conflicts of interest relevant to this arti- cle were reported.

H.M. has received honoraria for lectures from Astellas Pharma Inc., AstraZeneca, Dainippon Pharma Co, Eli Lilly, Kissei, Mitsubishi Tanabe Pharma Co., MSD, Novartis Pharma, Novo Nordisk Pharma and Sanofi; and received research funding from Astellas Pharma Inc., AstraZeneca, Daiichi Sankyo, Eli Lilly, Mitsubishi Tanabe Pharma Co., MSD, Novo Nordisk Pharma, Sanofi, Takeda Pharmaceutical Co., Ltd. and Taisho Toyama Pahrmaceutical Co., Ltd.

A.N. has received honoraria for lectures from Sanofi.

T.A. has received honoraria for lectures from Mitsubishi Tanabe Pharma Co., Chugai Pharmaceutical Co., Ltd., Astellas Pharma Inc., Takeda Pharmaceutical Co., Ltd., Pfizer Inc. and AbbVie Inc.; and received research funding from Astellas Pharma Inc., Takeda Pharmaceutical Co., Ltd., Mitsubishi Tanabe Pharma Co., Chugai Pharmaceutical Co., Ltd., Daiichi Sankyo Co. Ltd. and Otsuka Pharmaceutical Co., Ltd.

C.Y., Y.F., R.K., M.I., H.N. and H.K. declare no conflict of interest.

\section{References}

1. Heise T, Hermanski L, Nosek L, Feldman A, Rasmussen $\mathrm{S}$, et al. (2012) Insulin degludec: four times lower pharmacodynamic variability than insulin glargine under steady-state conditions in type 1 diabetes. Diabetes Obes Metab 14: 859-864.

2. Heise T, Pieber TR (2007) Towards peakless, reproducible and long-acting insulins. An assessment of the basal analogues based on isoglycaemic clamp studies. Diabetes Obes Metab 9: 648-659.

3. Ashwell SG, Gebbie J, Home PD (2006) Twice-daily compared with once-daily insulin glargine in people with Type 1 diabetes using meal-time insulin aspart. Diabet Med 23: 879-886.

4. Heise T, Nosek L, Bottcher SG, Hastrup H, Haahr H (2012) Ultra-long-acting insulin degludec has a flat and stable glucose-lowering effect in type 2 diabetes. Diabetes Obes Metab 14: 944-950.

5. Vijan S, Hayward RA, Ronis DL, Hofer TP (2005) Brief report: the burden of diabetes therapy: implications for the design of effective patient-centered treatment regimens. J Gen Intern Med 20: 479-482.

6. Heller S, Buse J, Fisher M, Garg S, Marre M, et al. (2012) Insulin degludec, an ultra-longacting basal insulin, versus insulin glargine in basal-bolus treatment with mealtime insulin aspart in type 1 diabetes (BEGIN Basal-Bolus Type 1): a phase 3, randomised, openlabel, treat-to-target non-inferiority trial. Lancet 379:
1489-1497.

7. Mathieu C, Hollander P, Miranda-Palma B, Cooper J, Franek E, et al. (2013) Efficacy and safety of insulin degludec in a flexible dosing regimen vs insulin glargine in patients with type 1 diabetes (BEGIN: Flex T1): a 26-week randomized, treat-to-target trial with a 26-week extension. J Clin Endocrinol Metab 98: 11541162.

8. Bode BW, Buse JB, Fisher M, Garg SK, Marre M, et al. (2013) Insulin degludec improves glycaemic control with lower nocturnal hypoglycaemia risk than insulin glargine in basal-bolus treatment with mealtime insulin aspart in Type 1 diabetes (BEGIN( $(\mathbb{R})$ Basal-Bolus Type 1): 2-year results of a randomized clinical trial. Diabet Med 30: 1293-1297.

9. Kusunoki Y, Katsuno T, Miyakoshi K, Ikawa T, Nakae R, et al. (2013) Effects of switching from insulin glargine or detemir to insulin degludec in patients with type 1 diabetes mellitus. Diabetes Ther 4:461-472.

10. Nakae R, Kusunoki Y, Katsuno T, Tokuda M, Akagami $\mathrm{T}$, et al. (2014) Medium-term effects of insulin degludec on patients with type 1 diabetes mellitus. Drugs RD 14: 133-138.

11. Taki K, Nishimura R, Morimoto A, Tsujino D, Miyashita Y, et al. (2010) Analysis of 24-hour glycemic excursions in patients with type 1 diabetes by using continuous glucose monitoring. Diabetes Technol Ther 12: 523-528. 
12. Su G, Mi SH, Li Z, Tao H, Yang HX, et al. (2013) Prognostic value of early in-hospital glycemic excursion in elderly patients with acute myocardial infarction. Cardiovasc Diabetol 12: 33.

13. Su G, Mi S, Tao H, Li Z, Yang H, et al. (2011) Association of glycemic variability and the presence and severity of coronary artery disease in patients with type 2 diabetes. Cardiovasc Diabetol 10: 19.

14. Muggeo M, Zoppini G, Bonora E, Brun E, Bonadonna RC, et al. (2000) Fasting plasma glucose variability predicts 10-year survival of type 2 diabetic patients: the Verona Diabetes Study. Diabetes Care 23: 45-50.

15. Rizzo MR, Marfella R, Barbieri M, Boccardi V, Vestini F, et al. (2010) Relationships between daily acute glucose fluctuations and cognitive performance among aged type 2 diabetic patients. Diabetes Care 33: 21692174.

16. Monnier L, Mas E, Ginet C, Michel F, Villon L, et al. (2006) Activation of oxidative stress by acute glucose fluctuations compared with sustained chronic hyperglycemia in patients with type 2 diabetes. JAMA 295: 16811687.

17. Chico A, Vidal-Rios P, Subira M, Novials A (2003) The continuous glucose monitoring system is useful for detecting unrecognized hypoglycemias in patients with type 1 and type 2 diabetes but is not better than frequent capillary glucose measurements for improving metabolic control. Diabetes Care 26: 1153-1157.

18. Cheyne EH, Cavan DA, Kerr D (2002) Performance of a continuous glucose monitoring system during controlled hypoglycaemia in healthy volunteers. Diabetes Technol Ther 4: 607-613.

19. Boyne MS, Silver DM, Kaplan J, Saudek CD (2003) Timing of changes in interstitial and venous blood glucose measured with a continuous subcutaneous glucose sensor. Diabetes 52: 2790-2794.

20. Molnar GD, Taylor WF, Ho MM (1972) Day-to-day variation of continuously monitored glycaemia: a further measure of diabetic instability. Diabetologia 8: 342-348.

21. Sakamoto M, Nishimura R, Irako T, Tsujino D, Ando K, et al. (2012) Comparison of vildagliptin twice daily vs. sitagliptin once daily using continuous glucose monitoring (CGM): crossover pilot study (J-VICTORIA study). Cardiovasc Diabetol 11: 92.

22. Jonassen I, Havelund S, Hoeg-Jensen T, Steensgaard DB, Wahlund PO, et al. (2012) Design of the novel protraction mechanism of insulin degludec, an ultra-longacting basal insulin. Pharm Res 29: 2104-2114.

23. Keating GM (2013) Insulin degludec and insulin degludec/insulin aspart: a review of their use in the management of diabetes mellitus. Drugs 73: 575-593. 